\title{
Commissioning of ALFABURST: initial tests and results
}

\author{
Kaustubh Rajwade ${ }^{1} *$, Jayanth Chennamangalam ${ }^{2}$, Duncan Lorimer ${ }^{1}$, Aris Karastergiou ${ }^{2}$, Dan \\ Werthimer $^{3}$, Andrew Siemion ${ }^{3}$, David MacMahon ${ }^{3}$, Jeff Cobb ${ }^{3}$, Christopher Williams ${ }^{2}$ and Wes \\ Armour $^{4}$ \\ ${ }^{1}$ Department of Physics and Astronomy, West Virginia University, \\ Morgantown, WV 26506, USA \\ *E-mail: kmrajwade@mix.wvu.edu \\ ${ }^{2}$ Astrophysics, University of Oxford, Denys Wilkinson building, \\ Keble Road, Oxford OX1 3RH, United Kingdom \\ ${ }^{3}$ Department of Astronomy, University of California Berkeley, \\ Berkeley, CA 94720, USA \\ ${ }^{4}$ Oxford e-Research Centre, University of Oxford, Keble Road, \\ Oxford OX1 3QG, United Kingdom
}

\begin{abstract}
Fast Radio Bursts (FRBs) are apparently one-time, relatively bright radio pulses that have been observed in recent years. The origin of FRBs is currently unknown and many instruments are being built to detect more of these bursts to better characterize their physical properties and identify the source population. ALFABURST is one such instrument. ALFABURST takes advantage of the 7-beam Arecibo L-band Feed Array (ALFA) receiver on the 305-m Arecibo Radio Telescope in Puerto Rico, to detect FRBs in real-time at L-band $(1.4 \mathrm{GHz})$. We present the results of recent on-sky tests and observations undertaken during the commissioning phase of the instrument. ALFABURST is now available for commensal observations with other ALFA projects.
\end{abstract}

Keywords: Fast Radio Bursts (FRBs); Instrumentation; Pulsars

\section{Introduction}

FRBs have been a mystery to astronomers since their discovery in $2007 \stackrel{2}{2}$. Since then, the number of confirmed detections has gone up to $\sim 15^{\underline{3}} \underline{\underline{6}}$. The origin of these bursts are still unknown. The dearth of discovery of these bursts has resulted in development of instruments at different radio telescopes capable of detecting short, sporadic radio pulses. Recent efforts in time-domain radio astronomy have focused on real-time FRB detection with the promise of rapid follow-up of new events. Here we present the results from the recent commissioning phase of one such instrument, ALFABURST which has been developed for the Arecibo Radio telescope to monitor the sky for FRBs in real time.

\section{Commissioning phase}

\subsection{Bandpass validation}

During the commissioning phase of the instrument, a number of validation tests were performed. One of them included an on-sky test to collect data using the instrument and generate a bandpass. The ALFABURST hardware acquired the 


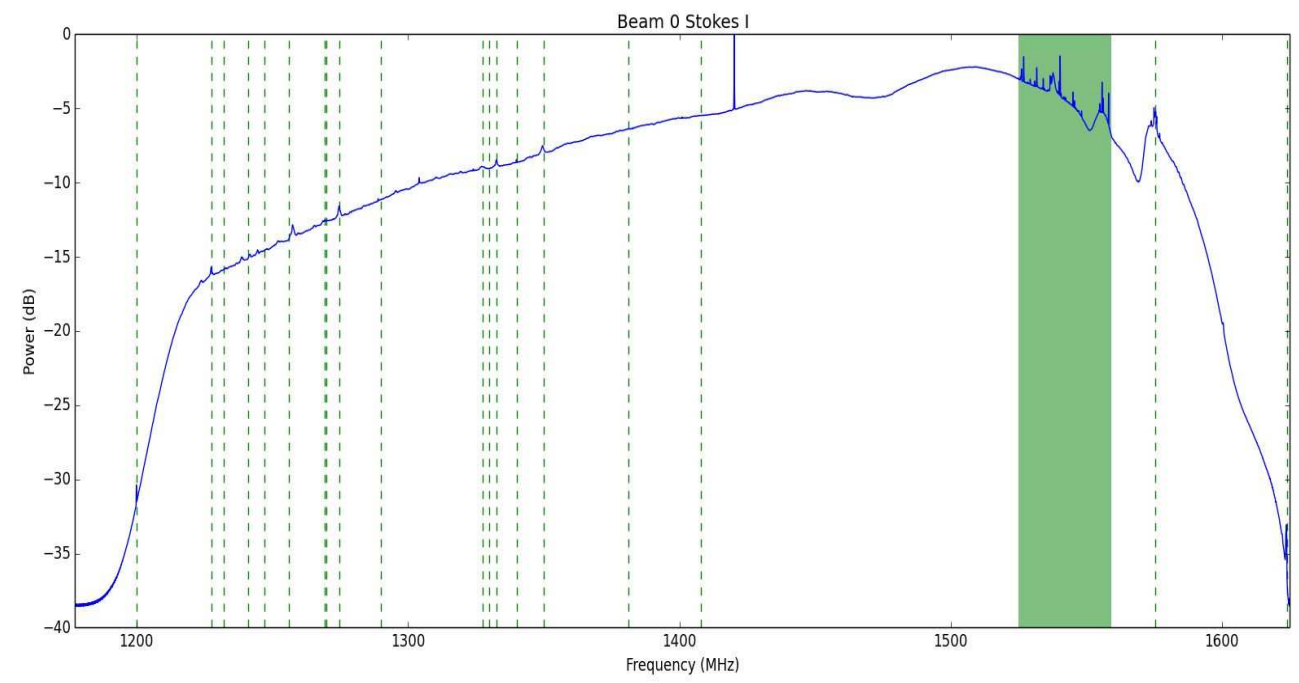

Fig. 1. Bandpass obtained from 1 of the 7 beams at L-band from a 5 minute observation using ALFABURST. The dashed vertical lines and the shaded region represent the known sources of RFI.

data from which the bandpass was obtained. The bandpass was also scrutinized to identify known sources of radio frequency interference (RFI). The peaks in the bandpass matched with the known RFI sources confirming that we obtained the correct band from the instrument (see Figure 1).

\subsection{Test observations}

After the initial tests were successful, we carried out observations with the instrument with dedicated time on the telescope. A few candidates (pulsars which emit bright pulses) were selected for the observation. ALFABURST looks for bright pulses in real-time using the ARTEMIS software pipeline ${ }^{1}$. We observed PSR B0611+22 and PSR B0531+21 (Crab Pulsar) in each of the beams of the ALFA receiver for a span of $\sim 2$ minutes. Fig. 2 shows the detection of bright pulses for pulsar PSR B0611+22 in all the seven beams of ALFA. The pulses are detected at the dispersion measure (DM) of PSR B0611+22 $\left(\sim 97 \mathrm{~cm}^{-3}\right.$ pc $)$. These observations were also beneficial for testing the beam to backend mapping of the instrument to make sure that we are detecting the pulsar in the expected data stream. We also tested the system in commensal mode to make sure that we're able to start the pipeline when ALFA is enabled and stop it when it's disabled.

\section{Conclusions and future work}

After successfully testing the instrument, ALFABURST is online to detect FRBs in real-time at Arecibo . Future work includes doing more observations to verify 


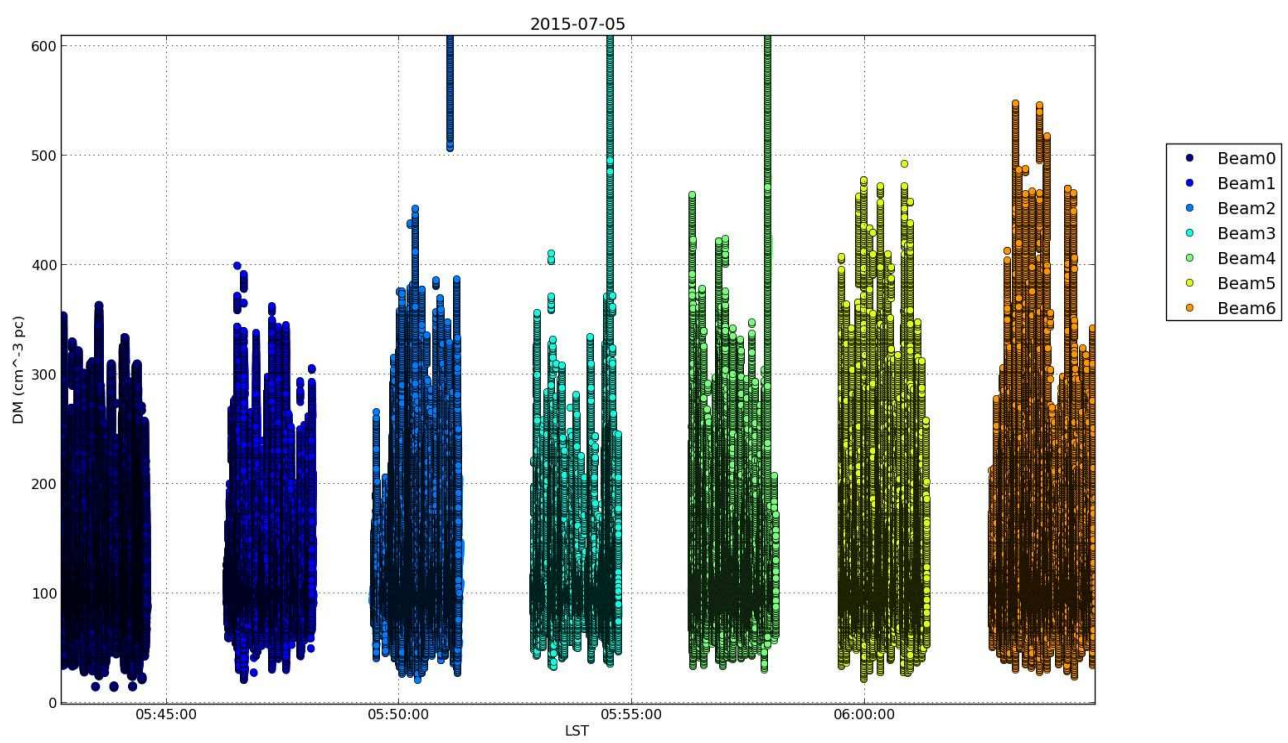

Fig. 2. DM versus time plot of detection of PSR B0611+22 in all the seven beams of the ALFA receiver.

if the whole pipeline is working smoothly over a wider bandwidth. Currently, we are able to process data in real-time over a bandwidth of $56 \mathrm{MHz}$ with a DM limit of $2560 \mathrm{~cm}^{-3} \mathrm{pc}$. Eventually, we plan on operating ALFABURST at the full bandwidth of $300 \mathrm{MHz}$ over a maximum DM of $\sim 10,000 \mathrm{~cm}^{-3} \mathrm{pc}$.

\section{References}

1. A. Karastergiou, et al., Limits on Fast Radio Bursts at $145 \mathrm{MHz}$ with ARTEMIS, a real-time software backend, arXiv:1506.03370, 2015.

2. D. R. Lorimer, et al., A Bright Millisecond Radio Burst of Extragalactic Origin, Sci., 318, 777, 2007.

3. E. Petroff, et al., A real-time fast radio burst: polarization detection and multiwavelength follow-up, Mon. Not. R. Astron. Soc., 447, 246, 2015.

4. L. G. Spitler, et al., Fast Radio Burst Discovered in the Arecibo Pulsar ALFA Survey, Astrophys. J., 790, 101, 2014.

5. D. Thornton, et al., A Population of Fast Radio Bursts at Cosmological Distances, Sci., 341, 53, 2013.

6. D. J. Champion, et al., Five nw Fast Radio Bursts from the HTRU high latitude survey: first evidence for two-component burst, ArXiv e-prints, 2015 